\title{
Facial Asymmetry as an Indicator of Psychological, Emotional, and Physiological Distress
}

\author{
Todd K. Shackelford and Randy J. Larsen \\ University of Michigan
}

\begin{abstract}
Fluctuating asymmetry (FA) is deviation from bilateral symmetry in morphological traits with asymmetry values that are normally distributed with a mean of 0 . FA is produced by genetic or environmental perturbations of developmental design and may play a role in human sexual selection. K. Grammer and R. Thornhill (1994) found that facial FA negatively covaries with observer ratings of attractiveness, dominance, sexiness, and health. Using self-reports, observer ratings, daily diary reports, and psychophysiological measures, the authors assessed the relationship between facial FA and health in 2 samples of undergraduates $(N=101)$. Results partially replicate and extend those of K. Grammer and R. Thornhill (1994) and suggest that facial FA may signal psychological, emotional, and physiological distress. Discussion integrates the authors' findings with previous research on FA and suggests future research needed to clarify the role of FA in human sexual selection.
\end{abstract}

Fluctuating asymmetry (FA) is random deviation from perfect bilateral symmetry in morphological traits for which the population mean asymmetry value is zero and the population distribution of signed asymmetry values approaches normality (Van Valen, 1962). A growing body of evidence ( see reviews in Gangestad, Thornhill, \& Yeo, 1994, and Thornhill \& Gangestad, 1993 ) indicates that FA manifests when the phenotypic expression of some developmental design is upset. FA increases in the presence of genetic disturbances such as deleterious recessives (Lerner, 1954; Parsons, 1990) and with homozygosity (Lerner, 1954). FA also increases with exposure to environmental perturbations during development such as extreme temperatures and pollutants (Parsons, 1990) and parasites (Gangestad et al., 1994; Thornhill \& Gangestad, 1993 ).

Variation in FA is perhaps best explained as a consequence of individual variation in ability to successfully resist or withstand various developmental disruptions, genetic or environmental (Thornhill \& Gangestad, 1994). Across a range of species, FA is correlated with lowered survival and growth rates and with diminished offspring production (Mitton \& Grant, 1984; Palmer \& Strobeck, 1986; Parsons, 1990; Thomhill, 1992a, 1992b; Thornhill \& Sauer, 1992).

Bilateral symmetry of morphological traits is positively correlated with genetic diversity, or heterozygosity, in many species,

Todd K. Shackelford and Randy J. Larsen, Department of Psychology, University of Michigan.

Data collection for this study was supported in part by Research Scientist Development Award KO1-MH00704 and Grant RO1-MH42057 from the National Institute of Mental Health. This article was prepared while Todd K. Shackelford was a Jacob K. Javits Graduate Fellow. We thank Jody Randall and Stephen Owens for help with data collection, coding, and entry.

Correspondence concerning this article should be addressed to Todd K. Shackelford, who is now at the Department of Psychology, University of Texas, 330 Mezes Hall, Austin, Texas 78712. Electronic mail may be sent via the Internct to shackelford@psy.utexas.edu. including humans (Thornhill \& Gangestad, 1993). Heterozygosity may be an important defense against parasitic infection in the context of the parasite-host coevolutionary process (Hamilton \& Zuk, 1982; Tooby, 1982; also see the review by Thornhill \& Gangestad, 1993). Thus, bilateral symmetry may be a manifestation of underlying heterozygosity and concomitant resistance to debilitating pathogens. Parasite-resistant and (hence) bilaterally symmetrical organisms are relatively more successful in intersexual and intrasexual competition for mates (Møller \& Pomiankowski, 1993; Thornhill, 1992a, 1992b; Thomhill \& Gangestad, 1993; Watson \& Thornhill, 1994). Consistent with "good genes" hypotheses of sexual selection, a meta-analysis of 26 published studies across 13 species indicates that FA is moderately heritable (Møller \& Thornhill, in press). Prospective mates may not be the only consumers of FA advertisements. Thornhill and Gangestad (1993) speculated that, insofar as symmetry signals general vigor, health, and other such qualities, humans may select friends in part based on facial and somatic symmetry. Humans may dispense nepotism in disproportionate amounts to their more symmetrical-and hence healthier-relatives. Thornhill and Gangestad (1993) speculated that, all else equal, a healthy friend or relative is a better investment than is an unhealthy friend or relative.

Recent evidence suggests an important role for FA in human sexual selection. In a study of university students, Gangestad et al. (1994) found a negative correlation between FA in seven heritable, nonfacial body characteristics and facial attractiveness after controlling for a variety of potentially confounding factors. This relationship reached statistical significance for men, but not women (see Gangestad et al., 1994, for possible explanations of this discrepancy). Further evidence that sexual selection favors developmental stability (as indicated by the absence of FA) in humans was found by Thornhill and Gangestad (1994). Those researchers found a negative relationship between the same measure of nonfacial body FA and self-reported lifetime number of sexual partners and a positive relationship between FA and selfreported age at first copulation. These relationships remained 
statistically significant after removing the effects of age, marital status, body height, ethnicity, physical anomalies associated with early prenatal development, and facial attractiveness.

Grammer and Thornhill (1994) wondered whether various bilaterally symmetrical human facial characteristics also might function as indicators of developmental stability or parasite resistance. If facial asymmetry reveals pathogenic disruption of developmental design and hence a lower quality immune system, individuals with greater facial FA are likely to be judged less physically attractive and ultimately will fare worse in intersexual and intrasexual competition. Grammer and Thornhill scored facial photographs along several dimensions of symmetry and then collected ratings by opposite-sex individuals of the faces depicted in the photographs along the dimensions of attractiveness, dominance, sexiness, and health. Grammer and Thornhill found, as predicted, that men and women whose faces displayed greater symmetry were rated by opposite-sex individuals as more attractive, more dominant, sexier, and healthier.

Although consistent with the notion that facial symmetry cues health, vigor, parasite resistance, and general genetic and developmental stability, Grammer and Thornhill's (1994) method of investigation provides only a weak test of the hypothesized cue value of facial symmetry. That is, Grammer and Thornhill's work suggests that people with more symmetrical faces are judged by others as more attractive, dominant, sexy, and healthy. Are they really though? Is facial symmetry, in fact, a cue to health, vigor, and developmental stability? Are people who have greater facial symmetry actually more physiologically healthy? More dominant? And if more facially symmetrical people are physiologically healthier, might they also be psychologically and emotionally healthier? The psychological and emotional health of a mate or friend is arguably as important as, if not more important than, his or her physical condition. To our knowledge, no previous research has examined the actual psychological, emotional, or physical health correlates of facial symmetry.

In this article we present analyses conducted using data from two independent groups of university students (67 women and 34 men in total). Each student completed daily measures of his or her psychological, emotional, and physical status over a 2 month period. In addition, students' general physiological fitness was objectively assessed via cardiac recovery time after a period of vigorous exercise. A head-and-shoulders photograph of each participant was taken and rated by independent groups of raters along various dimensions, including several personality dimensions, activity level, and attractiveness. Finally, each photograph was scored for asymmetries along five dimensions. The goals of this study were (a) to replicate Grammer and Thornhill's (1994) findings that more facially symmetrical people are rated as more attractive, more dominant, and more healthy; (b) to extend their findings by testing whether individuals displaying greater facial asymmetry are given less desirable ratings along several other dimensions; and (c) to investigate whether those exhibiting greater facial asymmetry actually report more frequent and more severe psychological, emotional, and physiological problems.

A strength of the current study is that we moved beyond social judgments of people with symmetrical and asymmetrical faces and examined actual data obtained from people who were rated on facial asymmetry. Another strength of this investigation is that many variables were available for examination, including measures obtained using several methods. Finally, the data set included an experiential sampling component, which allowed us to investigate naturalistic daily behaviors, emotions, and physical symptoms that may covary with facial asymmetry ratings.

In this study we drew on two archival data sets, in which many variables were available on two groups of intensively assessed participants. The fact that the data files on these participants contained their photographs gave us the opportunity to assess facial asymmetry and obtain observer ratings. Admittedly, the idea for a study of facial asymmetry came after the original data sets were collected, and so we had no input on what variables were obtained. Nevertheless, the sheer volume of data obtained on each participant allowed us to select many variables for analysis that were directly relevant to our hypotheses. In addition, many other variables were examined in a relatively exploratory manner. Because so little is known about the actual subject characteristics associated with facial asymmetry, we felt that such exploratory analyses were warranted. Whenever possible, however, we tested for replication across the two samples.

\section{Method}

\section{Participants}

Two samples of students were involved in this investigation. Sample 1 included 41 female and 16 male students (aged 18-23 years, $M=$ $\sim 20$ years) enrolled in a semester-long independent study course at a large midwestern university. Sample 2 included 26 female and 18 male students with a similar age structure as Sample 1. Sample 2 participants were enrolled in a semester-long independent study course at a different midwestern university. Individuals in both samples received credit toward their grades based on participation in weekly class meetings, the completion of assignments, and the writing of a final term paper.

\section{Materials and Procedure}

Personality and psychological variables. Participants in both samples completed a variety of standard personality measures over the course of the semester. These inventories are established in the literature and exhibit acceptable levels of reliability and validity. The students in Sample 1 completed the following measures: the Beck Depression Inventory (BDI; Beck, 1967); the Eysenck Personality Questionnaire (EPQ; Eysenck, Eysenck, \& Barrett, 1985); the Life Orientation Test (LOT; Scheier \& Carver, 1985); the Repression-Sensitization Scale (RS; Byrne, 1964); the Narcissistic Personality Inventory (NPI; Raskin \& Hall, 1979); Emotionality, Activity Level, Sociability, Impulsivity (EASI 3; Buss \& Plomin, 1975); the Emotional Control Questionnaire (ECQ; Roger \& Nesshover, 1987); the Taylor Manifest Anxiety Scale (MAS; Taylor, 1953); Augmenting - Form G2 (Herzog, Williams, \& Weintraub, 1984); the Locus of Control Scale (LOC; Phares, 1976); and the Brief Symptom Inventory (Derogatis \& Melisaratos, 1983).

Students in Sample 2 completed the following inventories: the EPQ (Eysenck et al., 1985); the EASI 3 (Buss \& Plomin, 1975); the ECQ (Roger \& Nesshover, 1987); Augmenting-Form G2 (Herzog et al., 1984); the LOC (Phares, 1976); and the Minnesota Multiphase Personality Inventory (MMPI; Hathaway \& McKinley, 1942).

Daily reports. Participants in each sample completed a report of daily activities, moods, and physical symptomatology. Identical copies of this form were completed two times daily for a period of 4 consecutive weeks during the regular academic semester. Each student completed one form at the midpoint of his or her day, reporting on his or her 
experiences during the first half of the day. A second report was completed close to the students' normal bedtime, which covered the second half of the day. Accurate recording of daily experiences was emphasized by the experimenters. Students were told that any form not completed within a 2-hr window of time was to be discarded. Daily reports were collected by experimenters daily. As the students began the daily phase of the study, the experimenters had established good rapport with them, resulting in excellent compliance with the daily reporting task (i.e., less than $10 \%$ of the daily reports were missing).

Students in Sample 1 reported the relative amount of time they spent in several daily situations, including time spent in typical (vs. novel) activities, time spent alone (vs. in social situations), and time spent in serious (vs. playful) activities.

Students in Sample 1 rated 40 mood adjectives selected to represent all octants of the circumplex model of emotion (Larsen \& Diener, 1992). The students' emotional experiences were rated on a 7-point Likert scale ranging from 0 (not at all) to 6 (extremely much). Students were provided with a written definition of each mood adjective (taken from Merriam-Webster's New Collegiate Dictionary) for daily referral.

Students in both samples used a checklist to indicate the occurrence of a variety of physical symptoms. For Sample 1, this checklist included headaches, trouble concentrating, runny nose, stomach or gastrointestinal trouble, muscle aches, sore throat or coughs, backaches, and feeling jittery. For Sample 2, a slightly different list of symptoms was used. Participants indicated the degree to which they had trouble getting to sleep the previous night $(0=$ not at all, $4=$ extremely). Additionally, Sample 2 participants reported how much they had eaten that morning $(0=$ nothing at all, $\mathbf{4}=$ more than a normal meal $)$. The symptom checklist used by Sample 2 participants included headaches, trouble concentrating, runny nose or congestion, nausea or upset stomach, muscle soreness, sore throat, backaches, nervousness, shortness of breath, and crying or the urge to cry. Students in Sample 1 also completed the Brief Symptom Inventory (Derogatis \& Melisaratos, 1983).

Cardiovascular fitness. Information on cardiovascular fitness was collected on participants from both samples via measurements of cardiac recovery time. On arriving at the laboratory, participants read a consent form informing them that exercise at moderate exertion would be required and inquiring about various health conditions that could be aggravated by participating in the study. Students with any of these conditions were not allowed to participate in the study. No student reported any medical condition that would put him or her at risk during exertion, and all students consented to participate.

To assess aerobic fitness in terms of cardiac recovery time, it was necessary to elevate participants' heart rates. Sample 1 participants rode a bicycle ergometer for $1 \mathrm{~min}$ at a moderately high exertion level (maintaining a speed of $11 \mathrm{mph}$ at a tension setting sufficient to expend 245 W of energy). Individuals in Sample 2 quickly stepped up and down a 2 -ft $(0.61-\mathrm{m})$-tall step for $1 \mathrm{~min}$. Both procedures resulted in at least 30 -bpm increases in heart rate.

Heart rate was monitored on a Grass Model 7D polygraph. A photoplethysmograph was attached to the participant's thumb to monitor the pulse wave. Signals were routed to a Grass 7P4 cardiotachometer to detect the rising slope of each pulse wave, with the Schmitt trigger adjusted to record heart rate for each participant in beats per minute. Continuous output was obtained during the entire exercise and recovery session by having the heart rate signal recorded on a moving strip chart.

After the 1-min exertion, participants were seated in a comfortable chair and allowed to recover fully. During this period, heart rate was continuously monitored. In Sample 1, aerobic fitness was assessed in terms of the slope of the cardiac recovery curve (Boutcher, 1990). Heart rates were averaged every $15 \mathrm{~s}$ for $6 \mathrm{~min}$. A linear regression was fit to the resulting 24 data points, and the slope of the regression line was calculated. A steep regression slope indicated faster recovery time, and hence better aerobic fitness, than a less steep slope. For Sample 2, aerobic fitness was assessed in terms of the time it took a participant's heart rate to return to his or her baseline level (Knapik, Jones, Reynolds, \& Staab, 1992). Because aerobic fitness was assessed with a different metric in each sample, we standardized fitness scores within each sample before conducting the analyses.

Observer ratings of photographs. A head-and-shoulders color photograph was taken of all Sample 1 participants and all but one of the Sample 2 participants. Participants were not given any special instructions about facial expression, head orientation, or, for example, whether glasses should be worn for the photograph. Photographs were taken at approximately the same distance from each participant. The negatives were developed into $4 \times 6$ in. $(10.16 \times 15.24 \mathrm{~cm})$ color photographs and $1 \times 1$ in. $(2.54 \times 2.54 \mathrm{~cm})$ projectable color slides. The slides of Sample 1 participants were rated on several dimensions by an independent group of raters (18 men and 19 women; mean age $=\sim 19$ years) The raters participated in exchange for credit toward their grade in an introductory psychology course. The raters assessed the photographs of Sample 1 participants on the following bipolar dimensions: unhappyhappy, unattractive-attractive, sluggish-active, introverted-extraverted, unreliable-reliable, disagreeable-agreeable, emotionally stable-emotionally unstable, and unintelligent-intelligent. The first adjective in the pair was assigned a value of -4 , the second a value of 4 , with 0 defining the midpoint of the Likert scale separating the terms. The ratings were standardized across all participants in Sample 1.

A second group of raters ( 18 men and 40 women; mean age $=\sim 20$ years) assessed the slides of Sample 2 participants on the same dimensions as Sample 1 participants were rated along, with the exception of intelligence. The dimensions were rated in a bipolar format, and ratings were standardized across all Sample 2 participants.

A third group of raters (six men and nine women) rated Sample 1 and Sample 2 photographs in exchange for extra credit in an advanced psychology research methods course at a large midwestern university. This group of raters assessed the photographs on two dimensions using a 9-point Likert scale: "What is this person's facial expression?" $(-4$ $=$ full frown, $0=$ neutral expression, and $4=$ full smile) ; "How genuine or 'real' does this person's facial expression appear?' $(-4=$ completely false or fake, 4 = completely genuine or "real").

Facial asymmetry measurements. The only published system that we know of for scoring facial asymmetry is a computer program developed by Grammer and Thornhill (1994). We adapted their scoring system so that we could use it without the aid of a computer program. We first enlarged the $4 \times 6$ in. $(10.16 \times 15.24 \mathrm{~cm})$ photographs to $8 \frac{1}{2} \times$ 11 in. $(21.59 \times 27.94 \mathrm{~cm})$ via a standard color copier. One of us and a research assistant performed each of the following tasks jointly, such that each task required agreement between the two people before it was considered complete. We first placed a sheet of graph transparency paper cross-hatched with 20 squares per inch on top of the photograph such that a secondary horizontal axis was by definition aligned with the center of the left pupil, cutting simultaneously through the center of the right pupil. We defined the vertical axis as that line perpendicular to and intersecting at the calculated midpoint of the line anchored by the left and right pupil centers. We defined the primary horizontal axis as tangent to the lowermost point at the base of the chin (i.e., the plane defined by the structural merging of the left and right mandibles).

Using a water-soluble marker, we consensually marked bilateral points for the following features: outer eye (points at outer closure of each eye), inner eye (points at inner closure of each eye), nostril width (widest points at base of nose), cheekbone width (points marking most prominent outer point of right and left cheekbone), and jaw width (points marking most prominent outer point of right and left jaw). A horizontal asymmetry score was calculated for each of the five bilateral characteristics using the following method: (a) calculate the distance (in $1 / 20$-in. pixels) from the vertical axis to the right bilateral point of the feature; (b) calculate the distance from the vertical axis to the left 
bilateral point of the feature; (c) calculate the absolute difference between the first and second steps; and (d) divide the absolute difference defined in the third step by the average distance of the target feature from the primary horizontal axis. ${ }^{1}$ A vertical asymmetry score was also calculated for each of the five bilateral features. The vertical asymmetry score was calculated in a manner identical to the horizontal score, except that the primary horizontal axis served as the reference axis in place of the vertical axis. Next, we calculated the mean of the asymmetries along the primary horizontal axis to produce a composite horizontal asymmetry score. Similarly, we computed the mean of the asymmetries along the vertical axis to create a composite vertical asymmetry score. Finally, to create a total asymmetry score, we computed the mean of the horizontal and vertical asymmetry composites. All asymmetry calculations were independently figured by one of us and a research assistant. ${ }^{2}$ An excellent level of agreement was attained for calculation of the vertical asymmetry scores ( $r=.89, p<.001 ; n=98$ ), horizontal asymmetry scores $(r=.80, p<.001 ; n=98)$, and total asymmetry scores $(r=$ $.86, p<.001 ; n=98)$. The asymmetry scores used in all subsequent analyses were the means of the two raters.

\section{Results}

Tables 1 and 2 show the results of correlational analyses for Samples 1 and 2 , respectively. ${ }^{3}$ Before highlighting the important findings, we discuss why we split the results by sex and why we present the results for three (rather than two or one) measures of facial symmetry.

We analyzed the data by sex for theoretical reasons, based primarily on evolutionary psychological theory. In humans, females are the limiting reproductive resource insofar as they, not men, are responsible for the heaviest parental investment burden. The minimum parental investment required of women is a physically exhaustive 9-month gestation period, parturition, perhaps followed by several months or years of lactation. The minimum parental investment of men is a few moments of sexual intercourse culminating in a single ejaculate. This differential minimum parental investment has, over human evolutionary history, selected for relatively more "choosy" women, and relatively less "choosy" men, in terms of mate selection (Trivers, 1972, 1985). Competition among men for sexual access to women thus tends to be more intense than competition among women for sexual access to men (Daly \& Wilson, 1988; Trivers, 1972). Men who have the qualities that make them more successful intrasexual competitors will tend to gain access to women more often than men who have characteristics that do not improve their intrasexual competitive ability.

Gangestad (1993), Thornhill and Gangestad (1993, 1994), and others ( see Cronin, 1991) have argued that one quality or collection of qualities that might increase a man's intersexual and intrasexual success is good genes. Gangestad (1993) and Thornhill and Gangestad (1993, 1994) argued that one indicator of good genes is facial symmetry. Thus, over human evolutionary history, men with relatively greater facial symmetry may have fared better in intrasexual and intersexual competition, whether because their "better" genome made them more successful in male-male competition for access to females, or because men with better genotypes were more desirable mates to women who sought to provide future offspring with the "best" genes. The upshot is that sexual selection is likely to have exerted greater selective pressures on men relative to women over human evolutionary history. Any indicator of good genes, for example, is likely to affect women's mate choices more than men's, on average, and so it is reasonable to expect that facial asymmetry, for example, may provide relatively more important information when displayed by men. Thus, we have presented the results in Tables 1 and 2 for men and women separately as well as collapsed across men and women.

We present three measures of asymmetry-horizontal, vertical, and total--for two reasons. First, the only published study on facial asymmetry that we could find, Grammer and Thornhill (1994), presented results only for horizontal asymmetry, yet the authors gave no indication of why they did not also present results for vertical asymmetry. Indeed, there is no a priori theoretical reason to expect one measure of asymmetry to be a better measure than the other. For this second reason, we present results for both horizontal and vertical asymmetry, in addition to taking the mean of these two measures of asymmetry and calling this total asymmetry. The correlations between vertical and horizontal asymmetry (including predicted horizontal asymmetry values; see Footnote 1$)$ were $.56(p<.001 ; n=56)$ for Sample 1 and $.50(p<.001 ; n=42)$ for Sample 2 .

Although our investigation involved two independent samples of participants and three independent sets of observers, none of our samples exceeded 41 when analyses were broken down by sex of participant. At the risk of slightly increased Type I error, we consider the results of our correlational analyses in terms of effect size, in addition to implementing standard statistical interpretation logic. Each correlation can be considered an effect size in and of itself. For a bivariate correlation coefficient, an effect size provides information about the magnitude of the relationship between two variables in standard deviation units. An effect size therefore is free of sample size limitations that can obscure important results in a standard statistical interpretational framework. According to Cohen (1988), correlations

\footnotetext{
${ }^{1}$ In approximately $40 \%$ of the photographs, participants were turning their head enough that the calculation of horizontal asymmetries was contaminated. To solve this problem, we first conducted an independentmeans $t$ test confirming the equivalence of the mean vertical asymmetries of participants who did and did not turn their head in the photograph for both sets of calculations (one of ours and the research assistant's). Next, for both sets of calculations (one of ours and the research assistant's), we derived an equation predicting horizontal from vertical asymmetry using the photographs for which both asymmetries could be calculated, Vertical asymmetry was a significant predictor of horizontal asymmetry for these photographs: $\beta 8=.825$ and $.711, p s=.003$ and .006 , and $t \mathrm{~s}(50)=3.11$ and 2.84 , respectively, for one of us and the research assistant. Having already established the equivalence of the mean vertical asymmetry scores for head-turners and non-head-turners, we applied the regression equations created based on asymmetry calculations of the non-head-turners to predict vertical asymmetry scores for the head-turners. Using this process, we were able to supply horizontal and total asymmetry scores for photographs in which the participant had turned his or her head.

${ }^{2}$ Two photographs from Sample 2 could not be scored for asymmetry because of photographic underexposure.

${ }^{3}$ Before conducting parametric correlations, we assessed the normality of horizontal, vertical, and total asymmetry scores for both samples collapsed across sex as well as separately for men and women. For every score in every sample and subsample, asymmetry was normally distributed, as indicated by nonsignificant Kolmogorov-Smirnov $z$ statistics (all $p \mathrm{~s}>35$ ).
} 
Table 1

Correlations of Facial Asymmetry With Personality and Psychological, Emotional, and Physiological Variables, With How Time Spent, and With Observer Ratings of Photographs in Sample 1

\begin{tabular}{|c|c|c|c|c|c|c|c|c|c|}
\hline \multirow[b]{2}{*}{ Variable } & \multicolumn{3}{|c|}{ Horizontal asymmetry } & \multicolumn{3}{|c|}{ Vertical asymmetry } & \multicolumn{3}{|c|}{ Total asymmetry ${ }^{a}$} \\
\hline & Women & Men & Total & Women & Men & Total & Women & Men & Total \\
\hline \multicolumn{10}{|l|}{ Personality and psychological scales } \\
\hline BDI Depression & .06 & .11 & .07 & .09 & $.51 *$ & .20 & .09 & .33 & .15 \\
\hline EPQ Extraversion & .27 & -.17 & .10 & $.32 *$ & -.04 & .18 & $.32 *$ & -.12 & .15 \\
\hline EPQ Neuroticism & -.05 & .46 & .06 & -.15 & .40 & -.01 & -.11 & $49^{*}$ & .03 \\
\hline EPQ Psychoticism & .16 &.$- \overline{02}$ & .13 & .25 & .23 & $.27^{*}$ & .23 & .11 & .21 \\
\hline EPQ Lie & -.12 & -.25 & -.15 & -.23 & -.46 & $-.29 *$ & -.19 & -.39 & -.24 \\
\hline LOT Optimism & -.10 & -.20 & -.10 & .17 & $-.50^{*}$ & -.01 & .02 & -.38 & -.06 \\
\hline Repression-Sensitization & -.05 & $.61^{* *}$ & .13 & -.15 & $.56^{*}$ & .05 & -.11 & $.67 * *$ & .11 \\
\hline NPI Entitlement & .17 & $\overline{21}$ & .20 & .13 &.$\overline{12}$ & .14 & .17 & .19 & .20 \\
\hline NPI Leadership & .04 & -.25 & -.01 & .30 & .10 & .26 & .17 & -.10 & .12 \\
\hline NPI Superiority & .13 & -.48 & -.03 & .22 & $-.61^{* *}$ & -.03 & .19 & $-.62 * *$ & -.03 \\
\hline NPI Self-Admiration & -.12 & -.05 & -.07 & -.02 & $-.53^{*}$ & -.16 & -.09 & -.30 & -.13 \\
\hline NPI General Narcissism & .08 & -.14 & .05 & .21 & -.47 & .03 & .16 & $=.33$ & .05 \\
\hline EASI 3 Emotionality & -.03 & -.16 & .01 & .05 & .18 & .23 & .01 & $\overline{-.01}$ & -.04 \\
\hline EASI 3 Fear & $-.35^{*}$ & .09 & -.22 & -.23 & .11 & -.13 & $-.33^{*}$ & .11 & -.20 \\
\hline EASI 3 Anger & $\overline{.00}$ & -.11 & -.04 & .07 & 38 & .15 & $\overline{.03}$ & .12 & .05 \\
\hline EASI 3 Tempo & -.31 & -.24 & $-.29^{*}$ & -.03 & -.16 & -.06 & -.21 & -.23 & -.21 \\
\hline EASI 3 Vigor & -.10 & -.33 & -.19 & .09 & -.18 & -.01 & -.02 & -.30 & -.13 \\
\hline EASI 3 Sociability & .06 & -.27 & -.07 & .17 & -.21 & .02 & .12 & $\overline{-.28}$ & -.04 \\
\hline EASI 3 Inhibition & .26 & .31 & .22 & .18 & .43 & .22 & .26 & .42 & .25 \\
\hline EASI 3 Decision Time & .25 & .09 & .21 & $42 * *$ & $\overline{41}$ & $.41 * *$ & $.37 *$ &.$\overline{26}$ & $.33 * *$ \\
\hline EASI 3 Sensation Seeking & .23 & -.14 & .14 & 30 & $-\overline{.04}$ & .21 & .29 & -.11 & .19 \\
\hline EASI 3 Perseverance & .14 & .45 & $.27^{*}$ &.$\overline{13}$ & 46 & .26 & .16 & $.52 *$ & $.30 *$ \\
\hline EASI 3 Impulsivity & $.34^{*}$ & .42 & $.35 * *$ & $40^{* *}$ & $.70 * *$ & $47 * * *$ & $.42 * *$ & $62 * *$ & $46 * * *$ \\
\hline EASI 3 Activity & -.26 & $-\overline{34}$ & $-.29 *$ & .02 & -.20 & -.04 & -.16 & -.32 & -.21 \\
\hline EASI 3 General Emotionality & -.14 & $\overline{-.08}$ & -.15 & -.03 & .31 & .04 & -.10 & $\overline{.11}$ & -.08 \\
\hline ECQ Rehearsal & -.05 & 41 & .09 & -.09 & .42 & .07 & -.08 & .47 & .09 \\
\hline ECQ Emotional Inhibition & .21 &.$- \overline{08}$ & .15 & .00 & $-\overline{20}$ & -.04 & .13 & $-\overline{15}$ & .08 \\
\hline ECQ Anger Control & -.18 & .30 & -.11 & -.18 & -.28 & -.21 & -.20 & .05 & -.17 \\
\hline ECQ Benign Control & -.05 & -.08 & -.06 & -.17 & $-.58^{*}$ & $-.30^{*}$ & -.12 & -.37 & -.19 \\
\hline Taylor Manifest Anxiety & .00 & .39 & .12 & .10 & .64** & $.27^{*}$ & .05 & $.57 *$ & .21 \\
\hline Augmenting (Form G2) & .13 & $-.67 * *$ & -.05 & $33^{*}$ & -.46 & .14 & .25 & $=.66^{* *}$ & .04 \\
\hline Internal Locus of Control & .20 & $-.56^{*}$ & -.11 & $\overline{21}$ & $.56 *$ & -.11 & .23 & $=.64 * t$ & -.12 \\
\hline \multicolumn{10}{|l|}{ How time spent, mean over 2 months } \\
\hline Time spent in typical (vs. novel) activities & .11 & $.58^{*}$ & .24 & .17 & $.56^{*}$ & $.28 *$ & .15 & $.65^{* *}$ & $.29 *$ \\
\hline Time spent alone (vs. in social situations) & -.06 & .23 & .07 & -.23 & $.50^{*}$ & .05 & -.15 & .40 & .07 \\
\hline Time spent in serious (vs. playful) activities & -.17 & -.02 & -.12 & -.08 & $.54^{*}$ & .12 & -.15 & .27 & -.02 \\
\hline \multicolumn{10}{|l|}{ Emotions experienced, mean over 2 months } \\
\hline Envious & .15 & 46 & $.27 *$ & .14 & .19 & .16 & .17 & .39 & .25 \\
\hline Jealous & .25 &.$\overline{44}$ & $.32 *$ & .22 & .28 & .25 & .27 &.$\overline{42}$ & $.32 *$ \\
\hline Relaxed & .21 & .38 &.$\overline{28 *}$ & .22 & -.10 & .12 & .24 & .19 &.$\overline{23}$ \\
\hline \multicolumn{10}{|l|}{ Physiological complaints or events } \\
\hline Headaches & -.16 & -.01 & -.10 & .00 & .28 & .10 & -.10 & .14 & -.01 \\
\hline Runny or stuffy nose & .08 & -.06 & .05 & .19 & .02 & .13 & .15 & -.03 & .09 \\
\hline Upset stomach/Gl trouble & .06 & -.03 & .03 & .08 & .24 & .08 & .08 & .11 & .06 \\
\hline Muscle soreness, cramps, aches & .14 & -.12 & .09 & .25 & -.21 & .13 & .21 & -.19 & .12 \\
\hline Sore throat or cough & -.08 & -.12 & -.07 & .21 & -.12 & .11 & .05 & -.14 & .01 \\
\hline Trouble concentrating or loss of interest & .02 & .44 & .12 & .10 & .45 & .18 & .07 & $.50 *$ & .17 \\
\hline Backache & -.08 & $\overline{.00}$ & -.01 & .13 &.$- \overline{06}$ & .04 & .01 & $-\overline{.03}$ & .01 \\
\hline Jittery & -.01 & .28 & .08 & -.09 & .44 & .08 & -.05 & .40 & .09 \\
\hline Brief Symptom Inventory physical symptoms & -.32 & .36 & -.12 & -.05 & $\overline{71} * *$ & .17 & -.23 & $.59 *$ & .01 \\
\hline Cardiovascular fitness ${ }^{\mathrm{b}}$ & $\overline{-.17}$ & .22 & -.02 & -.02 & .02 & -.01 & -.12 & .15 & -.02 \\
\hline \multicolumn{10}{|l|}{ Observer ratings from photograph } \\
\hline Happiness & -.27 & .12 & -.14 & -.15 & -.16 & -.17 & -.25 & -.01 & -.18 \\
\hline Attractiveness & -.04 & -.08 & -.06 & .24 & $=-.37$ & .05 & .10 & -.24 & -.01 \\
\hline Activity level & -.27 & -.20 & -.24 & -.11 & $\overline{-.42}$ & -.23 & -.22 & -.34 & $-.27 *$ \\
\hline Extraversion & -.28 & .16 & -.14 & -.04 & $=-.34$ & -.15 & -.20 & -.08 & -.16 \\
\hline Conscientiousness & -.24 & $-.51^{*}$ & $-.36^{* *}$ & -.21 & $\overline{=.42}$ & $-.30^{*}$ & -.25 & $=.53 *$ & $=.38^{* *}$ \\
\hline
\end{tabular}

(table continues) 
Table 1 (continued)

\begin{tabular}{|c|c|c|c|c|c|c|c|c|c|}
\hline \multirow[b]{2}{*}{ Variable } & \multicolumn{3}{|c|}{ Horizontal asymmetry } & \multicolumn{3}{|c|}{ Vertical asymmetry } & \multicolumn{3}{|c|}{ Total asymmetry ${ }^{a}$} \\
\hline & Women & Men & Total & Women & Men & Total & Women & Men & Total \\
\hline Agreeableness & -.24 & -.24 & -.26 & -.17 & -.22 & -.20 & -.23 & -.26 & $-.26^{*}$ \\
\hline Emotional stability & -.27 & -.24 & -.23 & -.13 & -.42 & -.21 & -.24 & -.37 & -.25 \\
\hline Intelligence & -.26 & $-.67^{* *}$ & $-.40 * *$ & -.24 & -.40 & $-.30^{*}$ & -.28 & $-.63^{*} *$ & $-.40 * *$ \\
\hline Smiling & $-.34 *$ & .18 & -.19 & -.27 & $\frac{-.08}{-.08}$ & -.23 & $-.34 *$ & .07 & -.23 \\
\hline Genuineness of expression & -.28 & $=.32$ & -.22 & .04 & $-60^{* *}$ & -.08 & -.16 & $-.51^{*}$ & -.18 \\
\hline
\end{tabular}

Note. Data were based on the responses of 41 women and 16 men. Observer ratings were based on head-and-shoulders color photographs of primary participants and were standardized across primary participants. According to Cohen (1988), correlations between .10 and .29 represent small effect sizes, correlations between .30 and .49 represent medium effect sizes, and correlations equal to or greater than .50 represent large effect sizes. Correlations representing medium or large effect sizes are underlined. BDI = Beck Depression Inventory; EPQ = Eysenck Personality Questionnaire; LOT = Life Orientation Test; NPI = Narcissistic Personality Inventory; EASI = Emotionality, Activity Level, Sociability, Impulsivity; $\mathrm{ECQ}=$ Emotional Control Questionnaire.

* Total asymmetry is the mean of horizontal and vertical asymmetry.

${ }^{b}$ Cardiovascular fitness (CF) is the inverse of cardiac recovery time after a brief period of vigorous exercise. CF was standardized across all Sample 1 participants.

${ }^{*} p \leq .05 .{ }^{* *} p \leq .01 . \quad{ }^{* *} p \leq .001$, two-tailed.

between .10 and .29 represent small effect sizes, correlations between .30 and .49 represent medium effect sizes, and correlations between .50 and 1.0 represent large effect sizes. Correlations representing medium or large effect sizes are underlined in both tables.

We specifically hypothesized that the correlations between asymmetry scores and scores along the target variables would obtain such that greater asymmetry would be associated with poorer psychological, emotional, or physiological health. Because we hypothesized directionality, we had, statistically speaking, "earned" the right to use one-tailed significance tests. Because we were concerned about obtaining significant relationships by chance, however, we used two-tailed tests of significance, making it substantially more difficult to obtain significance, particularly in light of our relatively small sample sizes.

\section{Personality and Psychological Variables}

Sample 1 men with greater facial asymmetry tended to be more depressed than men with less facial asymmetry as measured by the BDI. This finding was not replicated in Sample 2, however, on examination of the MMPI-Depression correlations. More facially asymmetrical women in both samples tended to be more extraverted than less facially asymmetrical women, as measured by the EPQ. The results for Sample 2 offer some evidence that more facially asymmetrical men are less extraverted than less asymmetrical men. Sample 1 men with more facial asymmetry also were more neurotic than men with less asymmetry, as measured by the EPQ, although this result was not replicated for Sample 2 men. There was some indication that psychopathy was positively related to facial asymmetry for both men and women in Sample 1. Sample 2 men scoring higher on psychopathy tended, however, to be less facially asymmetrical than men scoring lower on that scale. Sample 1 asymmetrical men and women tended to score lower on the EPQ Lie scale than did more symmetrical participants, suggesting less concern for appearing socially desirable. This finding was weakly repli- cated for Sample 2 men and women, particularly with regard to vertical asymmetry.

Sample 1 men who displayed more facial asymmetry tended to be less optimistic than men with less asymmetry, as measured by the LOT. Additionally, less facially symmetrical men tended to be psychologically repressive relative to more symmetrical men. Sample 1 men displaying less facial symmetry tended to feel inferior to others, have less self-admiration, and were less narcissistic than more facially symmetrical men, as measured by the NPI.

As measured by the EASI 3, Sample 1 men with greater facial asymmetry were more angry, had a lower physiological tempo, were less vigorous, were less sociable, were more inhibited, spent less time making important decisions, were more obsessive, were much more impulsive, were less active, and were more emotional than were men with less facial asymmetry. Replicating results found for Sample 1 men, Sample 2 men exhibiting greater facial asymmetry tended to be more emotionally labile and more angry than less asymmetrical men. Sample 1 women exhibiting greater asymmetry were less fearful, had a lower physiological tempo, were somewhat more inhibited, took less time to make important decisions, were somewhat more likely to be sensation seekers, exhibited much more impulsivity, and were less active than were less asymmetrical women. Replicating the results of Sample 1, more facially asymmetrical Sample 2 women also tended to be less active than less asymmetrical women. Additionally, Sample 2 women showed a tendency to be more angry than more symmetrical women.

As assessed by the ECQ, less facially symmetrical Sample 1 men tended to engage in more emotional rehearsal or rumination and to display less effective benign emotional control than more symmetrical men. Sample 2 men with greater facial asymmetry tended to be less emotionally inhibited, but, contrary to Sample 1 men, tended to display greater benign control or inhibition of impulses, relative to men with less asymmetry. No replicable findings emerged for women in the two samples with regard to emotional control.

Sample 1 men exhibiting greater asymmetry reported higher 
Table 2

Correlations of Facial Asymmetry With Personality and Psychological, Emotional, and Physiological Variables, With Participation in Various Activities, and With Observer Ratings of Photographs in Sample 2

\begin{tabular}{|c|c|c|c|c|c|c|c|c|c|}
\hline \multirow[b]{2}{*}{ Variable } & \multicolumn{3}{|c|}{ Horizontal asymmetry } & \multicolumn{3}{|c|}{ Vertical asymmetry } & \multicolumn{3}{|c|}{ Total asymmetry ${ }^{a}$} \\
\hline & Women & Men & Total & Women & Men & Total & Women & Men & Total \\
\hline \multicolumn{10}{|l|}{ Personality and psychological scales } \\
\hline EPQ Extraversion & $.42^{*}$ & .07 & .22 & .16 & -.42 & -.09 & .32 & -.25 & .03 \\
\hline EPQ Neuroticism &.$- \overline{04}$ & -.20 & -.13 & .18 & -.09 & .05 & .06 & -.18 & -.07 \\
\hline EPQ Psychoticism & .04 & $=.42$ & -.14 & -.08 & -.18 & -.13 & -.01 & -.37 & -.16 \\
\hline EPQ Lie & .01 & $\overline{-.06}$ & -.08 & -.26 & -.25 & -.23 & -.11 & -.14 & -.16 \\
\hline EASI 3 Emotionality & .12 & .36 & .18 & .05 & 40 & .23 & .11 & .41 & .22 \\
\hline EASI 3 Fear & .02 & .06 & -.02 & -.18 & .14 & -.04 & -.08 & .10 & -.03 \\
\hline EASI 3 Anger & .17 & .20 & .23 & -.38 & .35 & .01 & -.06 & .28 & .17 \\
\hline EASI 3 Tempo & -.17 & -.14 & -.12 & -.07 & .27 & .10 & -.15 & .01 & -.05 \\
\hline EASI 3 Vigor & -.16 & .17 & .00 & $-.40^{*}$ & -.03 & -.07 & -.36 & .05 & -.05 \\
\hline EASI 3 Sociability & .14 & -.08 & .08 & .03 & .05 & .03 & $\overline{.12}$ & -.04 & .07 \\
\hline EASI 3 Inhibition & -.25 & -.20 & -.23 & -.02 & .12 & .05 & -.19 & -.09 & -.14 \\
\hline EASI 3 Decision Time & -.02 & -.23 & -.12 & .13 & .09 & .11 & .05 & -.13 & -.04 \\
\hline EASI 3 Sensation Seeking & -.10 & -.32 & -.18 & .29 & .02 & .18 & .07 & -.21 & -.05 \\
\hline EASI 3 Perseverance & .19 & $\overline{-.29}$ & -.13 & .00 & .03 & .02 & .14 & -.19 & -.09 \\
\hline EASI 3 Impulsivity & -.11 & $=.37$ & -.25 & .16 & .11 & .14 & .00 & -.22 & -.12 \\
\hline EASI 3 Activity & -.32 & $\overline{-.10}$ & -.12 & -.13 & .24 & .07 & -.29 & .03 & -.06 \\
\hline EASI 3 General Emotionality & .13 & .27 & .18 & -.23 & .39 & .09 & -.01 & .35 & .17 \\
\hline ECQ Rehearsal & .08 & .12 & .06 & -.01 & .09 & .04 & .05 & .12 & .06 \\
\hline ECQ Emotional Inhibition & -.11 & -.48 & -.30 & .00 & $-.57 *$ & -.20 & -.05 & $-.59 *$ & -.27 \\
\hline ECQ Anger Control & -.13 & .06 & -.13 & -.04 & -.15 & -.07 & -.11 & -.02 & -.12 \\
\hline ECQ Benign Control & .08 & .43 & .21 & -.35 & .08 & -.15 & -.11 & .33 & .08 \\
\hline Augmenting (Form G2) & .33 &.$- \overline{02}$ & .13 & $\overline{-.13}$ & $-.52 *$ & -.17 & .07 &.$- \overline{36}$ & -.06 \\
\hline Internal Locus of Control & .15 & -.14 & .03 & .01 & $-.47^{*}$ & -.24 & .08 & -.39 & -.16 \\
\hline MMPI Lie & .02 & .35 & .27 & .07 & $\overline{.14}$ & .10 & .05 & .30 & .23 \\
\hline MMPI Fake Good & .09 & .25 & .24 & .32 & .14 & .20 & .22 & .22 & .25 \\
\hline MMPI Hysteria & -.02 & -.35 & -.11 & .08 & -.12 & -.06 & .02 & -.29 & -.10 \\
\hline MMPI Depression & -.19 & .03 & -.03 & .18 & .07 & .12 & -.05 & .05 & .03 \\
\hline MMPI Hypochondriasis & .07 & -.38 & -.11 & .18 & -.16 & -.04 & .13 & -.33 & -.10 \\
\hline MMPI Psychopathic Deviance & .07 & $\overline{-.16}$ & .00 & .37 & -.23 & .10 & .23 & -.20 & .04 \\
\hline MMPI Masculinity/Femininity & .07 & .23 & .25 & .16 & .20 & .12 & .13 & .24 & .23 \\
\hline MMPI Paranoia & .09 & .24 & .22 & .13 & .28 & .20 & .13 & .28 & .24 \\
\hline MMPI Psychasthenia & .15 & .09 & .20 & .26 & .09 & .14 & .23 & .10 & .20 \\
\hline MMPI Schizophrenia & $40^{*}$ & .21 & .26 & .09 & 33 & 30 & .26 & 31 & $32 *$ \\
\hline MMPI Mania & $43^{*}$ & .02 & .21 & .05 & .48 & $.31^{*}$ & .24 & .34 & $.31^{*}$ \\
\hline MMPI Social Introversion & -.18 & .13 & -.06 & -.26 & .05 & -.11 & -.26 & .11 & $-\overline{.09}$ \\
\hline \multicolumn{10}{|c|}{$\begin{array}{l}\text { Physiological complaints or events, mean } \\
\text { over } 2 \text { months }\end{array}$} \\
\hline Trouble getting to sleep & .12 & .04 & .07 & .29 & -.11 & .12 & .22 & -.01 & .10 \\
\hline Trouble staying asleep & .20 & $.62 *$ & $.41^{* *}$ & .23 & .31 & .29 & .26 & 47 & $.38^{* *}$ \\
\hline Amount eaten & .17 & $\underline{45}$ & .28 & .25 & .25 & .28 & .26 & .35 & $32^{*}$ \\
\hline \multicolumn{10}{|c|}{$\begin{array}{l}\text { Physiological complaints or events, total } \\
\text { over } 2 \text { months }\end{array}$} \\
\hline Headaches & .02 & -.36 & -.17 & .19 & .02 & .11 & .10 & -.24 & -.07 \\
\hline Trouble concentrating & .07 & -.30 & -.07 & .35 & -.01 & .23 & .21 & -.21 & .05 \\
\hline Runny nose or congestion & -.05 & $\overline{-.12}$ & -.08 &.$- \overline{07}$ & .32 & .15 & -.07 & .05 & .01 \\
\hline Nausea or upset stomach & -.16 & -.38 & -.27 & .05 & $-\overline{01}$ & .01 & -.09 & -.27 & -.19 \\
\hline Muscle soreness & $46^{*}$ & .07 & .12 & .21 & -.32 & -.09 & 37 & -.20 & -.02 \\
\hline Sore throat & -.26 & -.17 & -.17 & -.05 & .13 & .04 & $-\overline{21}$ & -.07 & -.10 \\
\hline Backache & $.59 * *$ & -.02 & .07 & .06 & -.39 & -.22 & .33 & -.28 & -.13 \\
\hline Nervousness &.$\overline{17}$ & .00 & -.03 & .15 & $\overline{-.05}$ & .07 &.$\overline{19}$ & -.14 & .01 \\
\hline Shortness of breath & .01 & -.25 & -.10 & -.21 & $-.48 *$ & -.29 & -.15 & -.43 & -.25 \\
\hline Crying or urge to cry & $.42 *$ & .11 & .28 & $.45^{*}$ & $\overline{.01}$ & .15 & $.52 * *$ & .05 & .23 \\
\hline Cardiovascular fitness $^{\mathrm{h}}$ &.$- \overline{01}$ & -.29 & -.07 & .09 & -.06 & .01 & .03 & -.22 & -.04 \\
\hline \multicolumn{10}{|c|}{ Total activity participation over 2 months } \\
\hline Went on date & .32 & .02 & .20 & .38 & .12 & .22 & $.43^{*}$ & .09 & .24 \\
\hline Bicycle riding & $-\overline{38}$ & .03 & -.21 & $-\overline{48} * *$ & .19 & -.20 & $-\overline{53 * *}$ & .15 & -.24 \\
\hline Paint, draw, crafts & .02 & -.14 & -.08 & -.30 & -.45 & $-.37 *$ & -.23 &.- .37 & -.30 \\
\hline Worked at job & $-.46^{*}$ & -.04 & -.24 & $\overline{-.41} *$ & -.19 & -.28 & $-.51 * *$ & -.15 & $-.30^{*}$ \\
\hline Novelty of recent events ${ }^{e}$ & .25 & -.03 & .13 & .08 & $-.50^{*}$ &.- .12 & .17 & -.36 & $\overline{-.03}$ \\
\hline \multicolumn{10}{|l|}{ Observer ratings from photograph } \\
\hline Happiness & .17 & -.06 & -.01 & -.13 & .37 & .13 & .06 & .11 & .05 \\
\hline
\end{tabular}


Table 2 (continued)

\begin{tabular}{|c|c|c|c|c|c|c|c|c|c|}
\hline \multirow[b]{2}{*}{ Variable } & \multicolumn{3}{|c|}{ Horizontal asymmetry } & \multicolumn{3}{|c|}{ Vertical asymmetry } & \multicolumn{3}{|c|}{ Total asymmetry ${ }^{a}$} \\
\hline & Women & Men & Total & Women & Men & Total & Women & Men & Total \\
\hline Attractiveness & .03 & -.13 & -.11 & .00 & .27 & .12 & .02 & .01 & -.02 \\
\hline Activity level & .01 & -.12 & -.09 & -.08 & .38 & .15 & -.03 & .07 & .00 \\
\hline Extraversion & .17 & -.20 & -.08 & -.14 & .32 & .11 & .05 & -.01 & -.01 \\
\hline Conscientiousness & .09 & $.52 *$ & .22 & -.01 & .32 & .17 & .06 & $49 *$ & .23 \\
\hline Agreeableness & .15 & .16 & .05 & -.01 & .46 & .21 & .10 & .30 & .13 \\
\hline Emotional stability & .05 & .05 & .04 & -.11 & .45 & .15 & -.02 & .22 & .09 \\
\hline Smiling & .22 & -.05 & -.02 & -.09 & .40 & .16 & .11 & .13 & .06 \\
\hline Genuineness of expression & -.02 & .15 & .04 & -.18 & -.05 & -.10 & -.10 & .09 & -.02 \\
\hline
\end{tabular}

Note. Data were based on responses from 26 women and 18 men. According to Cohen (1988), correlations between .10 and .29 represent small effect sizes, correlations between .30 and .49 represent medium effect sizes, and correlations equal to or greater than .50 represent large effect sizes. Correlations representing medium or large effect sizes are underlined. EPQ = Eysenck Personality Questionnaire; EASI = Emotionality, Activity Level, Sociability, Impulsivity; ECQ = Emotional Control Questionnaire; MMPI = Minnesota Multiphasic Personality Inventory.

${ }^{a}$ Total asymmetry is the mean of horizontal and vertical asymmetry. ${ }^{b}$ Cardiovascular fitness $(\mathrm{CF})$ is the inverse of cardiac recovery time after a brief period of vigorous exercise. CF was standardized across all Sample 2 participants. ${ }^{\circ}$ Reported as mean atypicality rating over 2 -month period. $* p \leq .05 . \quad * * p \leq .01$.

levels of anxiety than did more symmetrical Sample 1 men, as assessed by the MAS. More facially asymmetrical men in both samples showed a tendency toward augmenting (introversion and withdrawal ) rather than reducing, whereas more asymmetrical women in both samples tended to engage in a reducing rather than an augmenting approach. Men in both samples who displayed greater facial asymmetry tended to maintain a more external locus of control, relative to men with less asymmetry.

Men and women in Sample 2, but not in Sample 1, completed the MMPI. Relative to men with less facial asymmetry, men with greater asymmetry tended to score higher on the Lie subscale, showed a tendency toward invalidity (elevated $F$ scale), were somewhat less hysterical and less hypochondriacal, showed a tendency toward less psychopathic deviance, were slightly more psychasthenic (fearful, ruminative, and agitated) and paranoid, and exhibited a strong tendency toward schizophrenia and mania. Relative to more facially symmetrical women, less symmetrical women showed some tendency to fake good, had a stronger tendency toward psychopathic deviance, tended to be somewhat more psychasthenic and, like their male counterparts, showed substantial tendencies toward schizophrenia and mania.

\section{How Time Was Spent}

Sample 1 men displaying greater facial asymmetry tended to spend more time involved in "typical" rather than novel, unique, and interesting activities than did less asymmetrical men. These same asymmetrical men also were much more likely to have spent time alone over the 2-month study period. Finally, Sample 1 men with greater facial asymmetry tended to spend more time in serious rather than playful activities. Replicating the results for Sample 1 men, Sample 2 men exhibiting greater facial asymmetry spent more time engaged in typical rather than novel activities than did men with less asymmetry. These men also were less likely to have spent time painting, drawing, or involved in various other crafts over the 2-month study period.

Relative to less asymmetrical women, women in Sample 2 who exhibited greater facial asymmetry tended to go on more dates; tended to spend less time bicycle riding, painting, drawing, and participating in other crafts; and tended to spend less time working at a job.

\section{Emotional Experiences}

Sample 1 men with greater facial asymmetry felt more envious and more jealous than more symmetrical men. Less symmetrical Sample 1 women also tended to experience more jealousy than facially symmetrical women over the 2 -month study period.

\section{Physiological Complaints}

Sample 1 men showing less facial symmetry were somewhat more likely to report headaches than more symmetrical men. Less symmetrical Sample 1 men were slightly more likely to complain of an upset stomach or gastrointestinal problems and were substantially more likely to have trouble concentrating, experience a loss of interest, and feel jittery (symptoms of depression and anxiety), relative to more symmetrical men. Corroborating this picture of facial asymmetry signaling physical illness in Sample 1 men, asymmetrical men scored much higher than symmetrical men on the Brief Symptom Inventory. Facial asymmetry also appeared to be indicative of physical distress in Sample 2 men. Relative to less asymmetrical men, Sample 2 men displaying greater asymmetry had more difficulty staying asleep and reported eating more than they considered normal. These same men were also somewhat more likely to complain of a runny nose and congestion. Finally, more facially asymmetrical Sample 2 men tended to have lower cardiovascular fitness than less asymmetrical men.

Women in Sample 1 who had a less symmetrical face tended to have poorer health than facially symmetrical women. Relative to more facially symmetrical women, less symmetrical women showed some tendency to more frequently have a runny nose or congestion problems. Less symmetrical women also complained more of muscle soreness, muscle cramps, and muscle aches than did more symmetrical women. Like their male counterparts, 
more asymmetrical Sample 1 women also scored higher on the Brief Symptom Inventory, relative to less asymmetrical women. A similar picture of poor health emerged for Sample 2 women exhibiting greater facial asymmetry. Less symmetrical women tended to have greater trouble getting to sleep and staying asleep, in addition to complaining that they ate more than they thought was normal. Facially asymmetrical women in Sample 2 complained more often than symmetrical women of having trouble concentrating, complained more of muscle soreness and backaches, and were much more likely to cry or have the urge to cry.

\section{Observer Ratings of Photographs}

Sample 1 men who displayed greater facial asymmetry were generally rated as less attractive, less active, less extraverted, less conscientious, less emotionally stable, and much less intelligent, relative to less asymmetrical men. Additionally, Sample 1 men exhibiting greater facial asymmetry were less likely to be smiling and were judged to be displaying a less genuine expression in their head-and-shoulders photograph. Observer ratings of Sample 2 men tended not to replicate the findings for Sample 1 men and, indeed, less symmetrical Sample 2 men were judged to be more conscientious than more symmetrical men.

Sample 1 women who displayed greater facial asymmetry were generally rated as less happy, less active, less extraverted, less conscientious, less emotionally stable, and much less intelligent than more symmetrical Sample 1 women. Sample 1 women exhibiting facial asymmetry were less likely to be smiling and were rated as displaying a less genuine expression in their headand-shoulders photograph. Observer ratings of Sample 2 women tended not to replicate the findings for Sample 1 women.

\section{Discussion}

The current investigation had three general goals: (a) to replicate Grammer and Thornhill's (1994) findings that facially asymmetrical individuals are judged to be less attractive, less dominant, and less healthy than facially symmetrical individuals; (b) to extend Grammer and Thornhill's findings by determining whether people with less symmetrical faces might be given less desirable ratings along various other personality and health dimensions, as judged from a head-and-shoulders photograph; and (c) to investigate whether facial asymmetry would serve as one of several possible indicators of psychological, emotional, and physiological distress. Toward these ends, we present correlational analyses conducted on data from two independent groups of university students. Participants completed various indexes of psychological, emotional, and physical health over a 2-month study period. Additionally, three independent groups of observers rated head-and-shoulders photographs of the primary participants along various personality, affective, and physical health dimensions.

Our findings suggest that facial asymmetry, although certainly not the only cue to a person's psychological, emotional, and physiological well-being, is clearly one such cue. More facially asymmetrical participants in our two independent samples report more psychological, affective, and physiological problems. In addition, more asymmetrical people in one sample were rated on the basis of a head-and-shoulders photograph as being less healthy in these core dimensions by three independent sets of observers.

Our results are consistent with an emerging body of data suggesting that FA tracks, and is a manifestation of, sexual selection in humans (see, e.g., Gangestad \& Thornhill, in press; Gangestad et al., 1994; Thornhill \& Gangestad, 1993, 1994). Gangestad et al. (1994), for example, documented that people evidencing greater asymmetry in seven nonfacial bilateral traits were rated as less facially attractive by an independent group of observers on the basis of a head-and-shoulders photograph. even after controlling for many potential confounds. Thornhill and Gangestad (1994), using the same measure of FA, found that more symmetrical people, particularly men, reported having sex earlier and with a greater number of partners. These re searchers further demonstrated that the relationships between FA and age at first copulation and number of lifetime partners remained even after partialing out several potential confounds.

In the only published study examining facial asymmetry that we could find, Grammer and Thornhill (1994) found evidence suggesting that several bilaterally symmetrical facial characteristics may function as reliable cues to an individual's developmental stability or parasite resistance. Grammer and Thornhill argued that facial symmetry - or the lack of facial asymmetry - is an "honest" advertisement of high immunocompetence or good genes, insofar as exposure to pathogens or environmental pollutants, for example, was countered by an effective immune system. To the extent that parasites or environmental poisons disrupt the developmental design of an individual by overtaxing his or her immune system, this will be evidenced in imperfections in otherwise bilaterally symmetrical facial and body characteristics.

Grammer and Thornhill (1994) found that men and women whose faces displayed less symmetry were rated by oppositesex raters as less attractive, less dominant, less sexy, and less healthy than were people exhibiting greater facial symmetry. Although consistent with the idea that facial symmetry honestly advertises health, vigor, parasite resistance, and developmental stability more generally, Grammer and Thornhill's method of investigation does not offer the strongest test of this idea. They tested whether observers would provide less favorable ratings of people exhibiting greater facial asymmetry. In our investigation, we sought to take Grammer and Thornhill's paradigm and results a step further.

Our results in part replicate the results of Grammer and Thornhill (1994) and in part do not. The ratings of men in orie of our samples replicated Grammer and Thornhill's finding that facially asymmetrical individuals are less physically attractive. We did not replicate the negative relationship between facial asymmetry and rated attractiveness for men in our other sample, nor did we replicate this relationship for women in either sample. Grammer and Thornhill, however, used only opposite-sex raters, whereas we allowed men and women to rate both men and women. Future researchers should attempt a replication of Grammer and Thornhill's findings for attractiveness using only opposite-sex raters. Replicating Grammer and Thornhill, albeit weakly, we found that people displaying less facial asymmetry were rated as more dominant (extraverted). Finally, if we consider activity level as one component of health, we replicated 
Grammer and Thornhill's finding that facially symmetrical people are rated as healthier than facially asymmetrical people on the basis of a head-and-shoulders photograph.

This article is an important contribution to the growing literature on the role of symmetry in human sexual selection. This study is especially critical insofar as there is only one other study in the literature examining the significance of facial asymmetry for human social perception. Most importantly, however, our study provides the first test (to our knowledge) of whether facial asymmetry might serve as a reliable indicator of psychological, emotional, and physiological illness. Facial asymmetry does appear to serve as at least one of several indicators of distress in these important domains. The most impressive findings are those that held across both samples. Men in both samples displaying greater facial asymmetry were, for example, more depressed, more emotionally labile, and, with reference to vertical asymmetry, more impulsive than men with less facial asymmetry. Women in both samples displaying greater facial vertical asymmetry were, for example, more impulsive than women with less facial asymmetry. Women in both samples with greater facial asymmetry experienced more muscle soreness and cramping than did more symmetrical women.

Purusing Tables 1 and 2, it is evident that facial asymmetry signals poor psychological, emotional, and physiological health for both men and women. The relationships appear to be stronger for men, however. This suggests that facial asymmetry may be a more reliable signal of poor health when displayed by men relative to the signal value of facial asymmetry when displayed by women. This is consistent with the logic of parental investment theory (Trivers, 1972). In humans, females are the limiting reproductive resource. The minimum parental investment required of women is an energetically expensive 9-month gestation period, parturition, perhaps followed by several months or years of lactation. The minimum parental investment of men is a few moments of sexual intercourse and a single ejaculate. Over human evolutionary history, this differential minimum parental investment has selected for relatively more choosy women and relatively less choosy men in terms of mate selection (Trivers, 1972, 1985). Competition among women for sexual access to men tends to be less intense than competition among men for sexual access to women (Daly \& Wilson, 1988; Trivers, 1972). Men who have the qualities that make them intrasexual and intersexual winners will tend to gain access to women more often than men who have characteristics that render them intrasexual and intersexual losers.

Gangestad (1993) and Thornhill and Gangestad (1993, 1994) speculated that good genes might contribute to a man's relative intrasexual and intersexual success. Gangestad and Thornhill argued that one indicator of good genes is facial symmetry. Over human evolutionary history, men with relatively greater facial asymmetry might have fared worse in intrasexual and intersexual competition. This lower competitive ability may have been because their "bad genes" rendered them less capable in malemale competition or because men with "weak" genotypes were less desirable mates to women who sought to provide future offspring with the "best" genes. Sexual selection is likely to have exerted greater selective pressures on men relative to women. Any indicator of good genes, for example, is likely to affect women's mate choices more than men's, on average. We expect that facial asymmetry, for example, may provide more important information when displayed by men than women.

Our results are consistent with an emerging empirical literature indicating that asymmetry in various nonfacial traits is correlated with a variety of psychological and physiological illnesses (see Møller \& Thormhill, 1996, for a comprehensive review). To our knowledge, our study was the first to move beyond social judgments of people displaying particular facial characteristics. Although we do provide results pertaining to the social judgments made about men and women displaying symmetrical and asymmetrical faces, we also report results using data pertaining to the actual psychological health status, actual emotional health status, and actual physiological health status of people for whom we have facial asymmetry measurements.

Another strength of our investigation is embodied by our use of multiple methods. Rather than rely solely on one-shot selfreport, as is characteristic of most psychological research, participants in our study also completed daily reports of their emotional and physical well-being. We also collected extensive cardiovascular data on each participant to assess cardiovascular fitness. We took each participant's photograph and had these photographs rated along several health, personality, and emotional well-being dimensions by three independent groups of raters. Finally, rather than ask a new set of raters to provide gross judgments of facial asymmetry, we developed a scoring system to manually measure this multidimensional structural feature. The strengths of this article notwithstanding, we should point out several important limitations of our research that must be overcome in future investigations.

The most important limitation to our investigation is small sample size. Once analyses were broken up by sex of participant, we were left with no more than 41 participants per cell. It would be useful to include a more diverse group of participants in these and similar analyses. Our samples consisted largely of middle and upper class men and women attending prestigious American universities. The parasite theory of sexual selection should apply across cultures, and its hypotheses should be equally supported in samples of American university students and Zambian farmers.

Perhaps more germane to this investigation and research on whether facial asymmetry might serve as a reliable cue to psychological, emotional, and physiological distress is the specific manner in which facial asymmetry functions as an indicator of illness in these three important domains. What is the exact route by which facial asymmetry and psychological and behavioral impulsivity, for example, came to covary? What is the design of the psychological mechanisms that presumably have evolved in response to and to detect the presence of facial and body symmetry or lack thereof? Are there particular contexts in which facial asymmetry is more or less important? Is facial asymmetry ever irrelevant (perhaps when brought about by external trauma to the face, hence not providing a valid cue of developmental stability or immunocompetence)? A substantial amount of research must be done before researchers can make any definitive conclusions about the relevance of facial asymmetry in human sexual selection processes. The results of our investigation, along with the results provided by Grammer and Thornhill (1994), suggest that, at the very least, facial asymmetry is far 
from irrelevant and may in fact serve as a reliable cue to psychological, emotional, and physiological distress.

\section{References}

Beck, A. T. (1967). Depression: Clinical, experimental, and theoretical aspects. New York: Harper \& Row.

Boutcher, S. H. (1990). Aerobic fitness: Measurement and issues. Journal of Sport and Exercise Psychology, 12, 235-247.

Buss, A. H., \& Plomin, R. (1975). A temperament theory of personality. New York: Wiley.

Byrne, D. (1964). Repression-sensitization as a dimension of personality. Progress in Experimental Personality Psychology, 1, 169-220.

Cohen, J. (1988). Statistical power analysis for the behavioral sciences (2nd ed.). Hillsdale, NJ: Erlbaum.

Cronin, H. (1991). The ant and the peacock. Cambridge, England: Cambridge University Press.

Daly, M., \& Wilson, M. (1988). Homicide. Hawthorne, NY: Aldine de Gruyter.

Derogatis, L. R., \& Melisaratos, N. (1983). The Brief Symptom Inventory: An introductory report. Psychological Medicine, 13, 595-605.

Eysenck, S. B., Eysenck, H. J., \& Barrett, P. (1985). A revised version of the Psychoticism scale. Personality and Individual Differences, 6 , $21-29$.

Gangestad, S. W. (1993). Sexual selection and physical attractiveness: Implications for mating dynamics. Human Nature, 4, 205-235.

Gangestad, S. W., \& Thornhill, R. (in press). Human sexual selection and developmental stability. In J. A. Simpson \& D. T. Kenrick (Eds.), Evolutionary social psychology. Mahwah, NJ: Erlbaum.

Gangestad, S. W., Thornhill, R., \& Yeo, R. A. (1994). Facial attractiveness, developmental stability, and fluctuating asymmetry. Ethology and Sociobiology, 15, 73-85.

Grammer, K., \& Thornhill, R. (1994). Human facial attractiveness and sexual selection: The role of symmetry and averageness. Journal of Comparative Psychology, 108, 233-242.

Hamilton, W. D., \& Zuk, M. (1982). Heritable true fitness and bright birds: A role for parasites? Science, 218, 384-387.

Hathaway, S. R., \& McKinley, J. C. (1942). Minnesota Multiphasic Personality Inventory. Minneapolis: University of Minnesota Press.

Herzog, T. R., Williams, D. M., \& Weintraub, D. (1984). Meanwhile, back at personality ranch: The augmenters and reducers ride again. Journal of Personality and Social Psychology, 48, 1342-1352.

Knapik, J. J., Jones, B. H., Reynolds, K. L., \& Staab, J. S. (1992). Validity of self-assessed physical fitness. American Journal of Preventative Medicine, 8, 367-372.

Larsen, R. J., \& Diener, E. (1992). Promises and problems with the circumplex model of emotion. Review of Personality and Social Psychology, 13, 25-59.

Lerner, I. M. (1954). Genetic homeostasis. Edinburgh, Scotland: Oliver \& Boyd.

Mitton, J. B., \& Grant, M. C. (1984). Associations among protein heterozygosity, growth rate, and developmental homeostasis. Annual Review of Ecological Systems, 15, 497-499.
Møller, A. P., \& Pomiankowski, A. (1993). Fluctuating asymmetry and sexual selection. Genetica, $89,267-279$.

Møller, A. P., \& Thornhill, R. (in press). A meta-analysis of the heritability of developmental stability. Journal of Evolutionary Biology.

Møller, A. P., \& Thornhill, R. (1996). Developmental stability, disease, and medicine. Manuscript submitted for publication.

Palmer, A. R., \& Strobeck, C. (1986). Fluctuating asymmetry: Measurement, analysis, pattern. Annual Review of Ecology and Systematics, 17, 392-421.

Parsons, P. A. (1990). Fluctuating asymmetry: An epigenetic measure of stress. Biological Review; 65, 131-145.

Phares, E. J. (1976). Locus of control in personality. Morristown, NJ: General Learning Press.

Raskin, R. N., \& Hall, C. S. (1979). A narcissistic personality inventory. Psychological Reports, 45, 590

Roger, D., \& Nesshover, W. (1987). The construction and preliminary validation of a scale for measuring emotional control. Personality and Individual Differences, 8, 527-534.

Scheier, M. F., \& Carver, C. S. (1985). Optimism, coping, and health: Assessment and implications of generalized outcome expectancies. Health Psychology, 4, 219-247.

Taylor, J. (1953). A personality scale of manifest anxiety. Journal of Abnormal and Social Psychology, 48, 285-290.

Thornhill, R. (1992a). Fluctuating asymmetry and the mating system of the Japanese scorpionfly. Behavioral Ecology, 3, 277-283.

Thornhill, R. (1992b). Fluctuating asymmetry, interspecific aggression, and male mating tactics in two species of Japanese scorpionflies. Behavioral Ecology and Sociobiology, 30, 357-363.

Thornhill, R., \& Gangestad, S. W. (1993). Human facial beauty: Averageness, symmetry, and parasite resistance. Human Nature, 4, 237 269.

Thornhill, R., \& Gangestad, S. W. (1994). Fluctuating asymmetry and human sexual behavior. Psychological Science, 5, 297-302.

Thornhill, R., Gangestad, S. W., \& Comer, R. (1995). Human female orgasm and male fluctuating asymmetry. Animal Behavior, 50, 1601 1615 .

Thornhill, R., \& Sauer, K. P. (1992). Genetic size effects on the fighting ability of sons and daughters and mating success of sons in a scorpionfly. Animal Behaviour, 43, 255-264.

Tooby, J. (1982). Pathogens, polymorphism, and the evolution of sex. Journal of Theoretical Biology, 97, 557-576.

Trivers, R. L. (1972). Parental investment and sexual selection. In B. Camphell (Ed.), Sexual selertion and the descent of man (pp. 136179). New York: Aldine de Gruyter.

Trivers, R. (1985). Social evolution. Menlo Park, CA: Benjamin/ Cummings.

Van Valen, L. (1962). A study of fluctuating asymmetry. Evolution, 16, $125-142$.

Watson, P. J., \& Thornhill, R. (1994). Fluctuating asymmetry and sexual selection. Trends in Ecology and Evolution, 9, 21-25.

Received September 5, 1995

Revision received May 30, 1996

Accepted June 13, 1996 